Acta Crystallographica Section E

Structure Reports

Online

ISSN 1600-5368

\section{$N$-(2,4,6-Trimethylphenyl)succinamic acid}

\section{B. Thimme Gowda, ${ }^{a *}$ Sabine Foro, ${ }^{b}$ B. S. Saraswathi ${ }^{a}$ and Hartmut Fuess ${ }^{b}$}

a Department of Chemistry, Mangalore University, Mangalagangotri 574 199, Mangalore, India, and ${ }^{\mathbf{b}}$ Institute of Materials Science, Darmstadt University of Technology, Petersenstrasse 23, D-64287 Darmstadt, Germany

Correspondence e-mail: gowdabt@yahoo.com

Received 21 July 2009; accepted 27 July 2009

Key indicators: single-crystal X-ray study; $T=299 \mathrm{~K}$; mean $\sigma(\mathrm{C}-\mathrm{C})=0.003 \AA$; $R$ factor $=0.048 ; w R$ factor $=0.145 ;$ data-to-parameter ratio $=12.3$.

The amide bond in the title compound systematic name: 3-[(2,4,6-trimethylphenyl)aminocarbonyl]propionic acid\}, $\mathrm{C}_{13} \mathrm{H}_{17} \mathrm{NO}_{3}$, has a trans conformation. In the crystal, two molecules form a centrosymmetric dimer connected by pairs of $\mathrm{O}-\mathrm{H} \cdots \mathrm{O}$ hydrogen bonds. Intermolecular $\mathrm{N}-\mathrm{H} \cdots \mathrm{O}$ hydrogen bonds link the dimers into a three dimensional network.

\section{Related literature}

For related structures, see: Gowda et al. (2009a,b,c); Jagannathan et al. (1994). For the modes of interlinking carboxylic acids by hydrogen bonds, see: Leiserowitz (1976).<smiles>Cc1cc(C)c(NC(=O)CCC(=O)O)c(C)c1</smiles>

\section{Experimental}

\section{Crystal data}

$\mathrm{C}_{13} \mathrm{H}_{17} \mathrm{NO}_{3}$

$M_{r}=235.28$

Triclinic, $P \overline{1}$

$a=4.7646$ (4) $\AA$

$b=10.859(1) \AA$

$$
\begin{aligned}
& Z=2 \\
& \mathrm{Cu} K \alpha \text { radiation } \\
& \mu=0.72 \mathrm{~mm}^{-1}
\end{aligned}
$$

Data collection

Enraf-Nonius CAD-4 diffractometer

Absorption correction: $\psi$ scan (North et al., 1968)

$T_{\min }=0.692, T_{\max }=0.945$

3109 measured reflections

\section{Refinement}

$R\left[F^{2}>2 \sigma\left(F^{2}\right)\right]=0.048$

$w R\left(F^{2}\right)=0.145$

$S=1.05$

2234 reflections

182 parameters
$T=299 \mathrm{~K}$

$0.55 \times 0.25 \times 0.08 \mathrm{~mm}$

Table 1

Hydrogen-bond geometry $\left(\AA{ }^{\circ}\right)$.

\begin{tabular}{lllll}
\hline$D-\mathrm{H} \cdots A$ & $D-\mathrm{H}$ & $\mathrm{H} \cdots A$ & $D \cdots A$ & $D-\mathrm{H} \cdots A$ \\
\hline $\mathrm{N} 1-\mathrm{H} 1 N \cdots \mathrm{O} 1^{\mathrm{i}}$ & $0.86(2)$ & $2.10(2)$ & $2.9368(18)$ & $163.6(19)$ \\
$\mathrm{O} 2-\mathrm{H} 2 O \cdots \mathrm{O} 3^{\mathrm{ii}}$ & $0.88(3)$ & $1.80(3)$ & $2.679(2)$ & $172(3)$ \\
\hline
\end{tabular}

Symmetry codes: (i) $x-1, y, z$; (ii) $-x+2,-y+1,-z$.

Data collection: $C A D-4-P C$ (Enraf-Nonius, 1996); cell refinement: $C A D-4-P C$; data reduction: REDU4 (Stoe \& Cie, 1987); program(s) used to solve structure: SHELXS97 (Sheldrick, 2008); program(s) used to refine structure: SHELXL97 (Sheldrick, 2008); molecular graphics: PLATON (Spek, 2009); software used to prepare material for publication: SHELXL97.

BTG thanks the Alexander von Humboldt Foundation, Bonn, Germany, for a resumption of his research fellowship.

Supplementary data and figures for this paper are available from the IUCr electronic archives (Reference: BT5016).

\title{
References
}

Enraf-Nonius (1996). CAD-4-PC. Enraf-Nonius, Delft, The Netherlands.

Gowda, B. T., Foro, S., Saraswathi, B. S., Terao, H. \& Fuess, H. (2009a). Acta Cryst. E65, o399.

Gowda, B. T., Foro, S., Saraswathi, B. S., Terao, H. \& Fuess, H. (2009b). Acta Cryst. E65, o466.

Gowda, B. T., Foro, S., Saraswathi, B. S., Terao, H. \& Fuess, H. (2009c). Acta Cryst. E65, o873.

Jagannathan, N. R., Rajan, S. S. \& Subramanian, E. (1994). J. Chem. Crystallogr. 24, 75-78.

Leiserowitz, L. (1976). Acta Cryst. B32, 775-802.

North, A. C. T., Phillips, D. C. \& Mathews, F. S. (1968). Acta Cryst. A24, 351359.

Sheldrick, G. M. (2008). Acta Cryst. A64, 112-122.

Spek, A. L. (2009). Acta Cryst. D65, 148-155.

Stoe \& Cie (1987). REDU4. Stoe \& Cie GmbH, Darmstadt, Germany. 


\section{supporting information}

Acta Cryst. (2009). E65, o2056 [doi:10.1107/S1600536809029791]

\section{$N-(2,4,6-$ Trimethylphenyl)succinamic acid}

\section{B. Thimme Gowda, Sabine Foro, B. S. Saraswathi and Hartmut Fuess}

\section{S1. Comment}

The amide moiety is an important constituent of many biologically significant compounds. As a part of studying the effect of ring and side chain substitutions on the structures of this class of compounds (Gowda et al., 2009a,b,c), the crystal structure of $N$-(2,4,6-trimethylphenyl)-succinamic acid (I) \{systematic name: 3-[(2,4,6-trimethylphenyl)-aminocarbonyl]propionic acid $\}$ has been determined. The conformations of $\mathrm{N}-\mathrm{H}$ and $\mathrm{C}=\mathrm{O}$ bonds in the amide segment of the structure are trans to each other (Fig.1). But the conformations of the amide $\mathrm{O}$ atom and the carbonyl $\mathrm{O}$ atom of the acid segment are $c i s$ to each other Further, the conformations of the $\mathrm{C}(\mathrm{O})-\mathrm{C}$ bonds in the $\mathrm{N}-\mathrm{CO}-\mathrm{CH}_{2}-\mathrm{CH}_{2}-\mathrm{C}(\mathrm{O})-\mathrm{OH}$ segment have "trans" and "gauche" torsions with the adjacent $\mathrm{C}-\mathrm{H}$ bonds.

The $\mathrm{C}=\mathrm{O}$ and $\mathrm{O}-\mathrm{H}$ bonds of the acid group are in syn position to each other, similar to that observed in the crystal structures of $N$-(2,6-dimethylphenyl)- succinamic acid (Gowda et al., 2009b) and $N$-(2-chlorophenyl)succinamic acid (Gowda et al., 2009a) but in contrast to the anti positions observed in the structure of $N$-(3,5-dichlorophenyl)succinamic acid(Gowda et al., 2009c)

The torsional angles of the groups, $\mathrm{C} 1-\mathrm{N} 1-\mathrm{C} 7-\mathrm{C} 8, \mathrm{~N} 1-\mathrm{C} 7-\mathrm{C} 8-\mathrm{C} 9, \mathrm{C} 7-\mathrm{C} 8-\mathrm{C} 9-\mathrm{C} 10$ and $\mathrm{C} 8-\mathrm{C} 9-\mathrm{C} 10-\mathrm{O} 2$ in the side chain are $180.0(2)^{\circ},-160.5(2)^{\circ}, 63.5(2)^{\circ}$ and $-174.9(2)^{\circ}$, respectively. The $\mathrm{N}-\mathrm{H} \cdots \mathrm{O}$ and $\mathrm{O}-\mathrm{H} \cdots \mathrm{O}$ intermolecular hydrogen bonds pack the molecules in the structure into supramolecular chains (Table 1, Fig.2).

The modes of interlinking carboxylic acids by hydrogen bonds is described elsewhere (Leiserowitz, 1976). The packing of molecules involving dimeric hydrogen bonded association of each carboxyl group with a centrosymmetrically related neighbor has also been observed (Jagannathan et al., 1994).

\section{S2. Experimental}

The solution of succinic anhydride $(0.025 \mathrm{~mole})$ in toluene $(25 \mathrm{cc})$ was treated dropwise with the solution of 2,4,6-trimethylaniline $(0.025 \mathrm{~mole})$ also in toluene $(20 \mathrm{cc})$ with constant stirring. The resulting mixture was stirred for about one $\mathrm{h}$ and set aside for an additional hour at room temperature for the completion of reaction. The mixture was then treated with dilute hydrochloric acid to remove the unreacted 2,4,6-trimethylaniline. The resultant solid $N$-(2,4,6-trimethylphenyl)-succinamic acid was filtered under suction and washed thoroughly with water to remove the unreacted succinic anhydride and succinic acid. It was recrystallized to constant melting point from ethanol. The purity of the compound was checked by elemental analysis and characterized by its infrared spectra. The plate like colourless single crystals of the compound used in X-ray diffraction studies were grown in ethanolic solution by slow evaporation at room temperature.

\section{S3. Refinement}

$\mathrm{H}$ atoms bonded to $\mathrm{C}$ were positioned with idealized geometry using a riding model $[\mathrm{C}-\mathrm{H}=0.93 \AA$ to $0.97 \AA]$ with $U(\mathrm{H})$ set to $1.2 U_{\mathrm{eq}}(\mathrm{C})$. The other $\mathrm{H}$ atoms were located in a difference map and their position refined with $U(\mathrm{H})$ set to 
$1.2 U_{\text {eq }}(\mathrm{N}, \mathrm{O})$

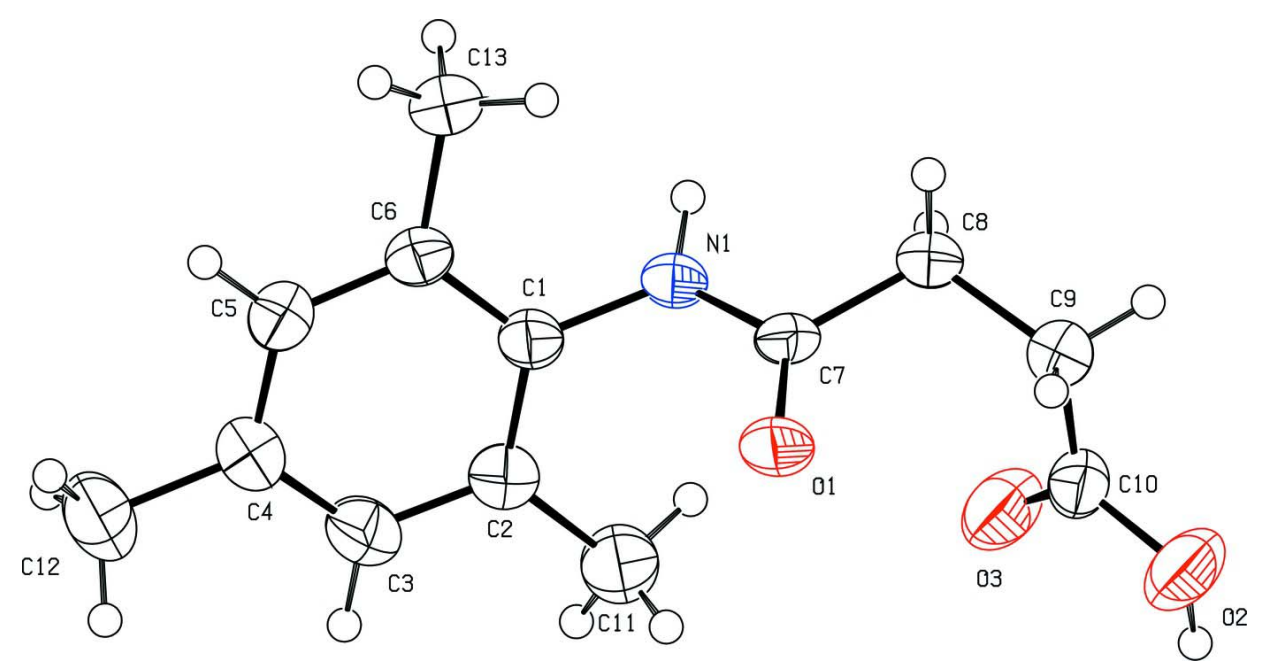

\section{Figure 1}

Molecular structure of (I), showing the atom labelling and the displacement ellipsoids are at the $50 \%$ probability level. The $\mathrm{H}$ atoms are represented as small spheres of arbitrary radii. 


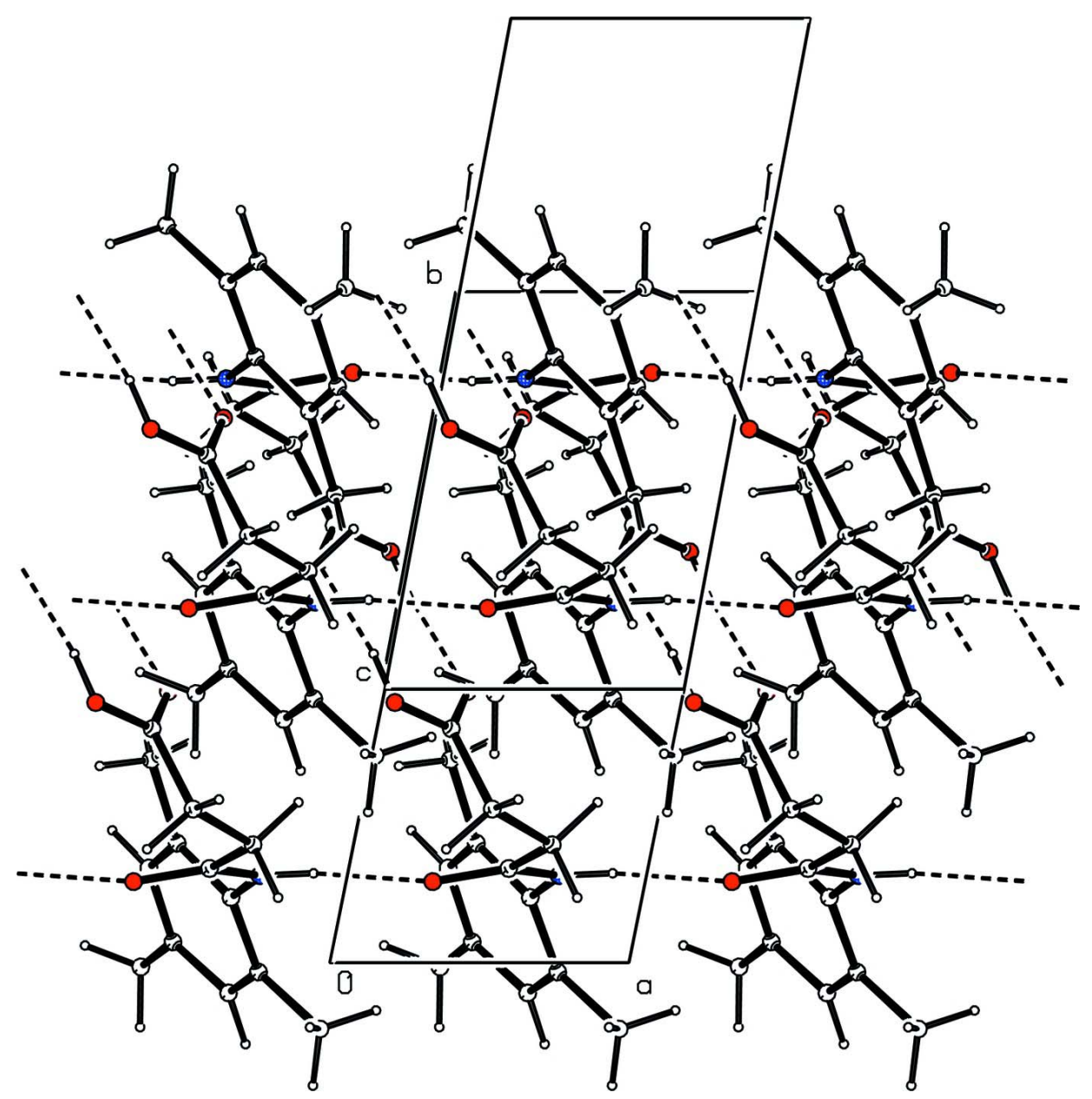

Figure 2

Molecular packing of (I) with hydrogen bonds shown as dashed lines.

\section{3-[(2,4,6-trimethylphenyl)aminocarbonyl]propionic acid}

\section{Crystal data}

$\mathrm{C}_{13} \mathrm{H}_{17} \mathrm{NO}_{3}$

$M_{r}=235.28$

Triclinic, $P \overline{1}$

Hall symbol: -P 1

$a=4.7646(4) \AA$

$b=10.859(1) \AA$

$c=13.111(2) \AA$

$\alpha=70.217(8)^{\circ}$

$\beta=86.158(8)^{\circ}$

$\gamma=79.351(8)^{\circ}$

$V=627.31(12) \AA^{3}$

\section{Data collection}

Enraf-Nonius CAD-4

diffractometer

Radiation source: fine-focus sealed tube

Graphite monochromator
$Z=2$

$F(000)=252$

$D_{\mathrm{x}}=1.246 \mathrm{Mg} \mathrm{m}^{-3}$

$\mathrm{Cu} K \alpha$ radiation, $\lambda=1.54180 \AA$

Cell parameters from 25 reflections

$\theta=4.4-22.9^{\circ}$

$\mu=0.72 \mathrm{~mm}^{-1}$

$T=299 \mathrm{~K}$

Plate, colourless

$0.55 \times 0.25 \times 0.08 \mathrm{~mm}$

$\omega / 2 \theta$ scans

Absorption correction: $\psi$ scan

(North et al., 1968)

$T_{\min }=0.692, T_{\max }=0.945$ 
3109 measured reflections

2234 independent reflections

1918 reflections with $I>2 \sigma(I)$

$R_{\text {int }}=0.014$

$\theta_{\max }=67.0^{\circ}, \theta_{\min }=3.6^{\circ}$

\section{Refinement}

Refinement on $F^{2}$

Least-squares matrix: full

$R\left[F^{2}>2 \sigma\left(F^{2}\right)\right]=0.048$

$w R\left(F^{2}\right)=0.145$

$S=1.05$

2234 reflections

182 parameters

0 restraints

Primary atom site location: structure-invariant direct methods

Secondary atom site location: difference Fourier map $h=-5 \rightarrow 2$

$k=-12 \rightarrow 12$

$l=-15 \rightarrow 15$

3 standard reflections every $120 \mathrm{~min}$

intensity decay: $1.0 \%$

Hydrogen site location: inferred from neighbouring sites

$\mathrm{H}$ atoms treated by a mixture of independent and constrained refinement

$w=1 /\left[\sigma^{2}\left(F_{\mathrm{o}}^{2}\right)+(0.0856 P)^{2}+0.1672 P\right]$ where $P=\left(F_{\mathrm{o}}^{2}+2 F_{\mathrm{c}}^{2}\right) / 3$

$(\Delta / \sigma)_{\max }=0.004$

$\Delta \rho_{\max }=0.30$ e $\AA^{-3}$

$\Delta \rho_{\text {min }}=-0.30$ e $\AA^{-3}$

Extinction correction: SHELXL97 (Sheldrick, 2008), $\mathrm{Fc}^{*}=\mathrm{kFc}\left[1+0.001 \times \mathrm{xc}^{2} \lambda^{3} / \sin (2 \theta)\right]^{-1 / 4}$

Extinction coefficient: 0.016 (3)

\section{Special details}

Geometry. All e.s.d.'s (except the e.s.d. in the dihedral angle between two 1.s. planes) are estimated using the full covariance matrix. The cell e.s.d.'s are taken into account individually in the estimation of e.s.d.'s in distances, angles and torsion angles; correlations between e.s.d.'s in cell parameters are only used when they are defined by crystal symmetry. An approximate (isotropic) treatment of cell e.s.d.'s is used for estimating e.s.d.'s involving 1.s. planes.

Refinement. Refinement of $F^{2}$ against ALL reflections. The weighted $R$-factor $w R$ and goodness of fit $S$ are based on $F^{2}$, conventional $R$-factors $R$ are based on $F$, with $F$ set to zero for negative $F^{2}$. The threshold expression of $F^{2}>\sigma\left(F^{2}\right)$ is used only for calculating $R$-factors (gt) etc. and is not relevant to the choice of reflections for refinement. $R$-factors based on $F^{2}$ are statistically about twice as large as those based on $F$, and $R$ - factors based on ALL data will be even larger.

Fractional atomic coordinates and isotropic or equivalent isotropic displacement parameters $\left(\AA^{2}\right)$

\begin{tabular}{lllll}
\hline & $x$ & $y$ & $z$ & $U_{\text {iso }} * / U_{\text {eq }}$ \\
\hline C1 & $0.3669(3)$ & $0.77823(17)$ & $0.30593(13)$ & $0.0376(4)$ \\
C2 & $0.5805(4)$ & $0.67491(18)$ & $0.36121(15)$ & $0.0452(4)$ \\
C3 & $0.6582(5)$ & $0.6700(2)$ & $0.46280(16)$ & $0.0549(5)$ \\
H3 & $0.804(5)$ & $0.598(2)$ & $0.5015(19)$ & $0.066^{*}$ \\
C4 & $0.5306(4)$ & $0.7610(2)$ & $0.51124(15)$ & $0.0512(5)$ \\
C5 & $0.3135(4)$ & $0.8597(2)$ & $0.45520(15)$ & $0.0469(5)$ \\
H5 & $0.225(5)$ & $0.923(2)$ & $0.4870(18)$ & $0.056^{*}$ \\
C6 & $0.2278(3)$ & $0.87030(18)$ & $0.35308(14)$ & $0.0400(4)$ \\
C7 & $0.4594(3)$ & $0.81334(16)$ & $0.11383(13)$ & $0.0368(4)$ \\
C8 & $0.3304(4)$ & $0.8203(2)$ & $0.00956(15)$ & $0.0494(5)$ \\
H8A & $0.214(5)$ & $0.912(2)$ & $-0.0210(18)$ & $0.059^{*}$ \\
H8B & $0.205(5)$ & $0.749(2)$ & $0.0314(18)$ & $0.059^{*}$ \\
C9 & $0.5540(4)$ & $0.8015(2)$ & $-0.07431(16)$ & $0.0543(5)$ \\
H9A & $0.683(5)$ & $0.867(2)$ & $-0.0887(19)$ & $0.065^{*}$ \\
H9B & $0.474(5)$ & $0.815(2)$ & $-0.142(2)$ & $0.065^{*}$ \\
C10 & $0.7408(4)$ & $0.6682(2)$ & $-0.04148(15)$ & $0.0479(5)$ \\
C11 & $0.7206(5)$ & $0.5687(2)$ & $0.31581(18)$ & $0.0607(6)$ \\
H11A & 0.8884 & 0.5946 & 0.2757 & $0.073^{*}$
\end{tabular}




$\begin{array}{lllll}\text { H11B } & 0.5898 & 0.5570 & 0.2686 & 0.073^{*} \\ \text { H11C } & 0.7730 & 0.4868 & 0.3741 & 0.073^{*} \\ \text { C12 } & 0.6208(6) & 0.7531(3) & 0.62154(18) & 0.0727(7) \\ \text { H12A } & 0.8203 & 0.7163 & 0.6316 & 0.087^{*} \\ \text { H12B } & 0.5120 & 0.6975 & 0.6763 & 0.087^{*} \\ \text { H12C } & 0.5875 & 0.8406 & 0.6270 & 0.087^{*} \\ \text { C13 } & -0.0127(4) & 0.9782(2) & 0.29629(16) & 0.0508(5) \\ \text { H13A } & -0.1896 & 0.9451 & 0.3122 & 0.061^{*} \\ \text { H13B } & 0.0210 & 1.0057 & 0.2195 & 0.061^{*} \\ \text { H13C } & -0.0227 & 1.0527 & 0.3210 & 0.061^{*} \\ \text { N1 } & 0.2852(3) & 0.78867(15) & 0.20030(11) & 0.0396(4) \\ \text { H1N } & 0.108(5) & 0.790(2) & 0.1887(16) & 0.047^{*} \\ \text { O1 } & 0.7050(2) & 0.83144(14) & 0.11738(10) & 0.0497(4) \\ \text { O2 } & 0.9467(3) & 0.65764(18) & -0.1116(13) & 0.0680(5) \\ \text { H2O } & 1.049(6) & 0.577(3) & -0.091(2) & 0.082^{*} \\ \text { O3 } & 0.7089(3) & 0.57886(15) & 0.04196(13) & 0.0631(4)\end{array}$

Atomic displacement parameters $\left(\AA^{2}\right)$

\begin{tabular}{lllllll}
\hline & $U^{11}$ & $U^{22}$ & $U^{33}$ & $U^{12}$ & $U^{13}$ & $U^{23}$ \\
\hline C1 & $0.0274(8)$ & $0.0490(9)$ & $0.0385(9)$ & $-0.0093(7)$ & $0.0005(6)$ & $-0.0158(7)$ \\
C2 & $0.0415(9)$ & $0.0474(9)$ & $0.0448(9)$ & $-0.0008(8)$ & $-0.0037(7)$ & $-0.0159(8)$ \\
C3 & $0.0538(12)$ & $0.0562(11)$ & $0.0483(11)$ & $0.0068(9)$ & $-0.0139(9)$ & $-0.0151(9)$ \\
C4 & $0.0503(11)$ & $0.0610(11)$ & $0.0425(10)$ & $-0.0052(9)$ & $-0.0069(8)$ & $-0.0188(8)$ \\
C5 & $0.0424(10)$ & $0.0570(11)$ & $0.0450(10)$ & $-0.0039(8)$ & $0.0014(8)$ & $-0.0244(9)$ \\
C6 & $0.0280(8)$ & $0.0502(10)$ & $0.0417(9)$ & $-0.0060(7)$ & $0.0020(6)$ & $-0.0159(7)$ \\
C7 & $0.0226(8)$ & $0.0466(9)$ & $0.0426(9)$ & $-0.0003(6)$ & $-0.0025(6)$ & $-0.0190(7)$ \\
C8 & $0.0281(9)$ & $0.0769(13)$ & $0.0448(10)$ & $0.0025(9)$ & $-0.0051(7)$ & $-0.0273(9)$ \\
C9 & $0.0417(10)$ & $0.0776(14)$ & $0.0417(10)$ & $0.0032(9)$ & $-0.0022(8)$ & $-0.0241(9)$ \\
C10 & $0.0373(9)$ & $0.0713(12)$ & $0.0450(10)$ & $-0.0081(8)$ & $0.0019(7)$ & $-0.0332(10)$ \\
C11 & $0.0688(14)$ & $0.0530(11)$ & $0.0553(12)$ & $0.0092(10)$ & $-0.0086(10)$ & $-0.0202(9)$ \\
C12 & $0.0836(17)$ & $0.0828(16)$ & $0.0519(12)$ & $0.0025(13)$ & $-0.0207(11)$ & $-0.0276(11)$ \\
C13 & $0.0356(10)$ & $0.0627(12)$ & $0.0517(10)$ & $0.0049(8)$ & $-0.0025(8)$ & $-0.0226(9)$ \\
N1 & $0.0213(7)$ & $0.0588(9)$ & $0.0420(8)$ & $-0.0074(6)$ & $-0.0022(6)$ & $-0.0206(7)$ \\
O1 & $0.0221(6)$ & $0.0789(9)$ & $0.0516(7)$ & $-0.0109(6)$ & $-0.0004(5)$ & $-0.0249(7)$ \\
O2 & $0.0600(10)$ & $0.0758(10)$ & $0.0631(9)$ & $0.0032(8)$ & $0.0198(7)$ & $-0.0280(8)$ \\
O3 & $0.0569(9)$ & $0.0678(9)$ & $0.0624(9)$ & $-0.0033(7)$ & $0.0161(7)$ & $-0.0256(8)$ \\
& & & & & &
\end{tabular}

Geometric parameters $\left(A,{ }^{\circ}\right)$

\begin{tabular}{llll}
\hline $\mathrm{C} 1-\mathrm{C} 6$ & $1.393(2)$ & $\mathrm{C} 8-\mathrm{H} 8 \mathrm{~B}$ & $1.02(2)$ \\
$\mathrm{C} 1-\mathrm{C} 2$ & $1.395(2)$ & $\mathrm{C} 9-\mathrm{C} 10$ & $1.491(3)$ \\
$\mathrm{C} 1-\mathrm{N} 1$ & $1.426(2)$ & $\mathrm{C} 9-\mathrm{H} 9 \mathrm{~A}$ & $0.99(3)$ \\
$\mathrm{C} 2-\mathrm{C} 3$ & $1.387(3)$ & $\mathrm{C} 9-\mathrm{H} 9 \mathrm{~B}$ & $0.94(3)$ \\
$\mathrm{C} 2-\mathrm{C} 11$ & $\mathrm{C} 10-\mathrm{O} 3$ & $1.215(2)$ \\
$\mathrm{C} 3-\mathrm{C} 4$ & $1.502(3)$ & $\mathrm{C} 10-\mathrm{O} 2$ & $1.311(2)$ \\
$\mathrm{C} 3-\mathrm{H} 3$ & $1.378(3)$ & $\mathrm{C} 11-\mathrm{H} 11 \mathrm{~A}$ & 0.9600 \\
$\mathrm{C} 4-\mathrm{C} 5$ & $0.96(2)$ & $\mathrm{C} 11-\mathrm{H} 11 \mathrm{~B}$ & 0.9600
\end{tabular}




\begin{tabular}{|c|c|c|c|}
\hline $\mathrm{C} 4-\mathrm{C} 12$ & $1.507(3)$ & $\mathrm{C} 11-\mathrm{H} 11 \mathrm{C}$ & 0.9600 \\
\hline $\mathrm{C} 5-\mathrm{C} 6$ & $1.387(2)$ & $\mathrm{C} 12-\mathrm{H} 12 \mathrm{~A}$ & 0.9600 \\
\hline $\mathrm{C} 5-\mathrm{H} 5$ & $0.94(2)$ & $\mathrm{C} 12-\mathrm{H} 12 \mathrm{~B}$ & 0.9600 \\
\hline $\mathrm{C} 6-\mathrm{C} 13$ & $1.506(2)$ & $\mathrm{C} 12-\mathrm{H} 12 \mathrm{C}$ & 0.9600 \\
\hline $\mathrm{C} 7-\mathrm{O} 1$ & $1.228(2)$ & $\mathrm{C} 13-\mathrm{H} 13 \mathrm{~A}$ & 0.9600 \\
\hline $\mathrm{C} 7-\mathrm{N} 1$ & $1.341(2)$ & $\mathrm{C} 13-\mathrm{H} 13 \mathrm{~B}$ & 0.9600 \\
\hline $\mathrm{C} 7-\mathrm{C} 8$ & $1.509(2)$ & $\mathrm{C} 13-\mathrm{H} 13 \mathrm{C}$ & 0.9600 \\
\hline $\mathrm{C} 8-\mathrm{C} 9$ & $1.517(3)$ & $\mathrm{N} 1-\mathrm{H} 1 \mathrm{~N}$ & $0.86(2)$ \\
\hline $\mathrm{C} 8-\mathrm{H} 8 \mathrm{~A}$ & $1.01(2)$ & $\mathrm{O} 2-\mathrm{H} 2 \mathrm{O}$ & $0.88(3)$ \\
\hline $\mathrm{C} 6-\mathrm{C} 1-\mathrm{C} 2$ & $121.06(15)$ & $\mathrm{C} 8-\mathrm{C} 9-\mathrm{H} 9 \mathrm{~A}$ & $110.4(14)$ \\
\hline $\mathrm{C} 6-\mathrm{C} 1-\mathrm{N} 1$ & $119.24(15)$ & $\mathrm{C} 10-\mathrm{C} 9-\mathrm{H} 9 \mathrm{~B}$ & $107.1(14)$ \\
\hline $\mathrm{C} 2-\mathrm{C} 1-\mathrm{N} 1$ & $119.69(15)$ & $\mathrm{C} 8-\mathrm{C} 9-\mathrm{H} 9 \mathrm{~B}$ & $112.8(15)$ \\
\hline $\mathrm{C} 3-\mathrm{C} 2-\mathrm{C} 1$ & $117.88(17)$ & $\mathrm{H} 9 \mathrm{~A}-\mathrm{C} 9-\mathrm{H} 9 \mathrm{~B}$ & $106(2)$ \\
\hline $\mathrm{C} 3-\mathrm{C} 2-\mathrm{C} 11$ & $119.76(17)$ & $\mathrm{O} 3-\mathrm{C} 10-\mathrm{O} 2$ & $123.15(19)$ \\
\hline $\mathrm{C} 1-\mathrm{C} 2-\mathrm{C} 11$ & $122.34(17)$ & $\mathrm{O} 3-\mathrm{C} 10-\mathrm{C} 9$ & $123.67(17)$ \\
\hline $\mathrm{C} 4-\mathrm{C} 3-\mathrm{C} 2$ & $122.86(18)$ & $\mathrm{O} 2-\mathrm{C} 10-\mathrm{C} 9$ & $113.17(18)$ \\
\hline $\mathrm{C} 4-\mathrm{C} 3-\mathrm{H} 3$ & $119.0(14)$ & $\mathrm{C} 2-\mathrm{C} 11-\mathrm{H} 11 \mathrm{~A}$ & 109.5 \\
\hline $\mathrm{C} 2-\mathrm{C} 3-\mathrm{H} 3$ & $118.1(14)$ & $\mathrm{C} 2-\mathrm{C} 11-\mathrm{H} 11 \mathrm{~B}$ & 109.5 \\
\hline $\mathrm{C} 3-\mathrm{C} 4-\mathrm{C} 5$ & $117.53(17)$ & $\mathrm{H} 11 \mathrm{~A}-\mathrm{C} 11-\mathrm{H} 11 \mathrm{~B}$ & 109.5 \\
\hline $\mathrm{C} 3-\mathrm{C} 4-\mathrm{C} 12$ & $121.54(19)$ & $\mathrm{C} 2-\mathrm{C} 11-\mathrm{H} 11 \mathrm{C}$ & 109.5 \\
\hline $\mathrm{C} 5-\mathrm{C} 4-\mathrm{C} 12$ & $120.93(19)$ & $\mathrm{H} 11 \mathrm{~A}-\mathrm{C} 11-\mathrm{H} 11 \mathrm{C}$ & 109.5 \\
\hline $\mathrm{C} 4-\mathrm{C} 5-\mathrm{C} 6$ & $122.27(17)$ & $\mathrm{H} 11 \mathrm{~B}-\mathrm{C} 11-\mathrm{H} 11 \mathrm{C}$ & 109.5 \\
\hline $\mathrm{C} 4-\mathrm{C} 5-\mathrm{H} 5$ & $118.5(14)$ & $\mathrm{C} 4-\mathrm{C} 12-\mathrm{H} 12 \mathrm{~A}$ & 109.5 \\
\hline $\mathrm{C} 6-\mathrm{C} 5-\mathrm{H} 5$ & $119.2(14)$ & $\mathrm{C} 4-\mathrm{C} 12-\mathrm{H} 12 \mathrm{~B}$ & 109.5 \\
\hline $\mathrm{C} 5-\mathrm{C} 6-\mathrm{C} 1$ & $118.35(16)$ & $\mathrm{H} 12 \mathrm{~A}-\mathrm{C} 12-\mathrm{H} 12 \mathrm{~B}$ & 109.5 \\
\hline $\mathrm{C} 5-\mathrm{C} 6-\mathrm{C} 13$ & $120.18(16)$ & $\mathrm{C} 4-\mathrm{C} 12-\mathrm{H} 12 \mathrm{C}$ & 109.5 \\
\hline $\mathrm{C} 1-\mathrm{C} 6-\mathrm{C} 13$ & $121.46(15)$ & $\mathrm{H} 12 \mathrm{~A}-\mathrm{C} 12-\mathrm{H} 12 \mathrm{C}$ & 109.5 \\
\hline $\mathrm{O} 1-\mathrm{C} 7-\mathrm{N} 1$ & $123.22(15)$ & $\mathrm{H} 12 \mathrm{~B}-\mathrm{C} 12-\mathrm{H} 12 \mathrm{C}$ & 109.5 \\
\hline $\mathrm{O} 1-\mathrm{C} 7-\mathrm{C} 8$ & $121.66(15)$ & $\mathrm{C} 6-\mathrm{C} 13-\mathrm{H} 13 \mathrm{~A}$ & 109.5 \\
\hline $\mathrm{N} 1-\mathrm{C} 7-\mathrm{C} 8$ & $115.11(14)$ & C6- $1313-\mathrm{H} 13 \mathrm{~B}$ & 109.5 \\
\hline $\mathrm{C} 7-\mathrm{C} 8-\mathrm{C} 9$ & $112.74(15)$ & $\mathrm{H} 13 \mathrm{~A}-\mathrm{C} 13-\mathrm{H} 13 \mathrm{~B}$ & 109.5 \\
\hline $\mathrm{C} 7-\mathrm{C} 8-\mathrm{H} 8 \mathrm{~A}$ & $106.9(13)$ & $\mathrm{C} 6-\mathrm{C} 13-\mathrm{H} 13 \mathrm{C}$ & 109.5 \\
\hline $\mathrm{C} 9-\mathrm{C} 8-\mathrm{H} 8 \mathrm{~A}$ & $107.8(13)$ & $\mathrm{H} 13 \mathrm{~A}-\mathrm{C} 13-\mathrm{H} 13 \mathrm{C}$ & 109.5 \\
\hline $\mathrm{C} 7-\mathrm{C} 8-\mathrm{H} 8 \mathrm{~B}$ & $105.4(13)$ & $\mathrm{H} 13 \mathrm{~B}-\mathrm{C} 13-\mathrm{H} 13 \mathrm{C}$ & 109.5 \\
\hline $\mathrm{C} 9-\mathrm{C} 8-\mathrm{H} 8 \mathrm{~B}$ & $112.3(13)$ & $\mathrm{C} 7-\mathrm{N} 1-\mathrm{C} 1$ & $123.64(14)$ \\
\hline $\mathrm{H} 8 \mathrm{~A}-\mathrm{C} 8-\mathrm{H} 8 \mathrm{~B}$ & $111.7(18)$ & $\mathrm{C} 7-\mathrm{N} 1-\mathrm{H} 1 \mathrm{~N}$ & $117.1(14)$ \\
\hline $\mathrm{C} 10-\mathrm{C} 9-\mathrm{C} 8$ & $114.18(18)$ & $\mathrm{C} 1-\mathrm{N} 1-\mathrm{H} 1 \mathrm{~N}$ & $118.7(14)$ \\
\hline $\mathrm{C} 10-\mathrm{C} 9-\mathrm{H} 9 \mathrm{~A}$ & $106.1(14)$ & $\mathrm{C} 10-\mathrm{O} 2-\mathrm{H} 2 \mathrm{O}$ & $111.1(18)$ \\
\hline $\mathrm{C} 6-\mathrm{C} 1-\mathrm{C} 2-\mathrm{C} 3$ & $-2.6(3)$ & $\mathrm{N} 1-\mathrm{C} 1-\mathrm{C} 6-\mathrm{C} 5$ & $-179.01(15)$ \\
\hline $\mathrm{N} 1-\mathrm{C} 1-\mathrm{C} 2-\mathrm{C} 3$ & $178.60(16)$ & $\mathrm{C} 2-\mathrm{C} 1-\mathrm{C} 6-\mathrm{C} 13$ & $-177.02(16)$ \\
\hline $\mathrm{C} 6-\mathrm{C} 1-\mathrm{C} 2-\mathrm{C} 11$ & $175.73(18)$ & $\mathrm{N} 1-\mathrm{C} 1-\mathrm{C} 6-\mathrm{C} 13$ & $1.8(2)$ \\
\hline $\mathrm{N} 1-\mathrm{C} 1-\mathrm{C} 2-\mathrm{C} 11$ & $-3.1(3)$ & $\mathrm{O} 1-\mathrm{C} 7-\mathrm{C} 8-\mathrm{C} 9$ & $20.7(3)$ \\
\hline $\mathrm{C} 1-\mathrm{C} 2-\mathrm{C} 3-\mathrm{C} 4$ & $1.1(3)$ & $\mathrm{N} 1-\mathrm{C} 7-\mathrm{C} 8-\mathrm{C} 9$ & $-160.51(18)$ \\
\hline $\mathrm{C} 11-\mathrm{C} 2-\mathrm{C} 3-\mathrm{C} 4$ & $-177.3(2)$ & $\mathrm{C} 7-\mathrm{C} 8-\mathrm{C} 9-\mathrm{C} 10$ & $63.5(3)$ \\
\hline $\mathrm{C} 2-\mathrm{C} 3-\mathrm{C} 4-\mathrm{C} 5$ & $0.9(3)$ & $\mathrm{C} 8-\mathrm{C} 9-\mathrm{C} 10-\mathrm{O} 3$ & $4.2(3)$ \\
\hline $\mathrm{C} 2-\mathrm{C} 3-\mathrm{C} 4-\mathrm{C} 12$ & $-179.8(2)$ & $\mathrm{C} 8-\mathrm{C} 9-\mathrm{C} 10-\mathrm{O} 2$ & $-174.88(17)$ \\
\hline
\end{tabular}




$\begin{array}{llll}\mathrm{C} 3-\mathrm{C} 4-\mathrm{C} 5-\mathrm{C} 6 & -1.3(3) & \mathrm{O} 1-\mathrm{C} 7-\mathrm{N} 1-\mathrm{C} 1 & -1.2(3) \\ \mathrm{C} 12-\mathrm{C} 4-\mathrm{C} 5-\mathrm{C} 6 & 179.3(2) & \mathrm{C} 8-\mathrm{C} 7-\mathrm{N} 1-\mathrm{C} 1 & 179.97(16) \\ \mathrm{C} 4-\mathrm{C} 5-\mathrm{C} 6-\mathrm{C} 1 & -0.2(3) & \mathrm{C} 6-\mathrm{C} 1-\mathrm{N} 1-\mathrm{C} 7 & 116.28(19) \\ \mathrm{C} 4-\mathrm{C} 5-\mathrm{C} 6-\mathrm{C} 13 & 179.05(18) & \mathrm{C} 2-\mathrm{C} 1-\mathrm{N} 1-\mathrm{C} 7 & -64.9(2) \\ \mathrm{C} 2-\mathrm{C} 1-\mathrm{C} 6-\mathrm{C} 5 & 2.2(3) & & \end{array}$

Hydrogen-bond geometry $\left(A,{ }^{\circ}\right)$

\begin{tabular}{lllll}
\hline$D-\mathrm{H} \cdots A$ & $D-\mathrm{H}$ & $\mathrm{H} \cdots A$ & $D \cdots A$ & $D-\mathrm{H} \cdots A$ \\
\hline $\mathrm{N} 1-\mathrm{H} 1 N^{\cdots} \cdots \mathrm{O} 1^{\mathrm{i}}$ & $0.86(2)$ & $2.10(2)$ & $2.9368(18)$ & $163.6(19)$ \\
$\mathrm{O} 2-\mathrm{H} 2 O \cdots 3^{\mathrm{ii}}$ & $0.88(3)$ & $1.80(3)$ & $2.679(2)$ & $172(3)$ \\
\hline
\end{tabular}

Symmetry codes: (i) $x-1, y, z$; (ii) $-x+2,-y+1,-z$. 